\title{
Médiévales
}

Langues, Textes, Histoire

74 | printemps 2018

Chanter la Croisade albigeoise

\section{Wendy Marie HOOFNAGLE, The Continuity of the \\ Conquest. Charlemagne and Anglo-Norman Imperialism}

Philadelphie, The Penn State University Press, 2016, 193 p.

\section{Fanny Madeline}

\section{(2) OpenEdition}

Journals

Édition électronique

URL : https://journals.openedition.org/medievales/8817

DOI : $10.4000 /$ medievales. 8817

ISSN : 1777-5892

Éditeur

Presses universitaires de Vincennes

Édition imprimée

Date de publication : 15 juillet 2018

Pagination : 185-189

ISBN : 978-2-84292-837-7

ISSN : 0751-2708

Référence électronique

Fanny Madeline, « Wendy Marie hoofnAgle, The Continuity of the Conquest. Charlemagne and Anglo-

Norman Imperialism », Médiévales [En ligne], 74 I printemps 2018, mis en ligne le 10 août 2018, consulté le 23 avril 2022. URL : http://journals.openedition.org/medievales/8817 ; DOI : https://doi.org/ 10.4000/medievales.8817

Ce document a été généré automatiquement le 23 avril 2022.

Tous droits réservés 


\title{
Wendy Marie HOOFNAGLE, The Continuity of the Conquest. Charlemagne and Anglo-Norman Imperialism
}

Philadelphie, The Penn State University Press, 2016, 193 p.

\author{
Fanny Madeline
}

\section{RÉFÉRENCE}

Wendy Marie HoOfNAGLE, The Continuity of the Conquest. Charlemagne and Anglo-Norman Imperialism, Philadelphie, The Penn State University Press, 2016, 193 p.

Le propos de W. M. Hoofnagle s'inscrit dans le courant des études se réclamant des théories " post-coloniales » appliquées au Moyen Âge, qui cherchent à comprendre la période à partir d'autres catégories que celles des " modernes", et notamment en faisant ressortir des phénomènes hybrides qui avaient été jusqu'alors ignorés ou négligés. Cette démarche, l'auteure entend l'appliquer pour comprendre comment la figure hybride de Charlemagne constitua un modèle de royauté idéale permettant aux Normands de légitimer leur projet impérial en Angleterre après 1066. Alors que l'histoire de la légende du saint empereur tout au long du Moyen Âge et au-delà constitue un champ de recherche depuis de nombreuses années, celui-ci s'est jusqu'à présent limité aux royautés capétiennes et allemandes, laissant de côté sa réception dans le monde anglo-normand. En réévaluant sa place dans la culture politique des Normands, cet ouvrage comble ainsi une lacune dans l'historiographie de la mémoire de Charlemagne. Mais si les études faisaient défaut, c'est sans doute autant par manque d'intérêt des historiens que par manque de documentation. Les traces explicites, qu'elles soient textuelles ou matérielles, ne sont pas très nombreuses, et cette rareté explique que le propos de l'auteure soit essentiellement un exercice d'interprétation. 
Son objectif vise cependant à interpréter autant les discours de revendication d'une continuité impériale carolingienne (très peu nombreux) que les pratiques à travers lesquelles les ducs de Normandie ont affirmé cette continuité jusqu'au XII siècle. Il s'agit donc d'un essai sur la mise en pratique de l'idéologie impériale carolingienne davantage qu'une étude des références explicites à la figure de Charlemagne comme modèle d'autorité, dans la mesure où ce deuxième objectif, qui est sans cesse recherché, est clairement le moins convainquant de l'étude. À travers l'analyse des pratiques, c'est l'influence que la mémoire de Charlemagne joua dans l'identité normande qui est traquée et la manière dont elle façonna leurs conceptions de l'autorité et de leurs relations avec les Anglais.

2 Partant du constat que l'idée d'empire ne fut pas homogène tout au long du Moyen Âge, l'auteure discute de la figure impériale de Constantin souvent évoquée comme symbole de la continuité impériale romaine en montrant qu'elle était beaucoup moins unanime que celle de Charlemagne, notamment du fait de la fameuse « donation » qui faisait des papes les véritables successeurs des empereurs romains. En tant qu'empereur résolument séculier, Charlemagne constituait, au contraire, un modèle de royauté beaucoup plus adapté à l'idéologie royale anglo-normande, comme il l'avait été aussi pour la royauté anglo-saxonne - dans la continuité de laquelle Guillaume le Conquérant voulait se placer. Les premiers ducs de Normandie se seraient ainsi considérés, du point de vue des pratiques gouvernementales, comme les véritables dépositaires de l'héritage carolingien, notamment après 987 . Cet héritage est essentiel pour comprendre la vision que les Normands avaient d'eux-mêmes comme les promoteurs dans le monde politique européen d'une société impériale urbaine et hautement sophistiquée. L'ouvrage se compose de trois grands chapitres centrés sur des pratiques : le premier est consacré aux « Politiques de conversion et l'idéologie de l'impérialisme »; le deuxième, intitulé " Faire sa marque : l'idéologie impériale de la topographie », analyse les pratiques de construction ; le dernier, " Apprivoiser les bêtes sauvages : un nouveau regard sur la New Forest ", fait de la chasse une pratique essentiellement carolingienne.

3 Le premier chapitre part de l'idée que l'empire de Charlemagne se détachait du modèle romain en le surpassant. Charlemagne offrait non seulement l'image du chef militaire triomphateur, à l'instar des Césars, mais aussi d'un chef spirituel qui chercha tout au long de son règne à convertir les peuples païens au christianisme. Cette politique de conversion, qui justifiait la sacralité de sa royauté, constituait, selon l'auteure, le cœur de l'idéologie impériale carolingienne. Or, cette politique de conversion, appelée aussi soft power, se retrouve dans la politique impériale de Guillaume le Conquérant et de ses successeurs, investis d'une mission de civilisation des peuples barbares des marges celtiques. En regardant ce phénomène, connu également sous le concept "d'européanisation » de la Grande-Bretagne, comme un projet impérial d'unification des peuples normands et anglais, l'auteure propose une lecture politique de ce qui a longtemps été considéré comme un phénomène essentiellement culturel, et s'inscrit ainsi dans la continuité de la démarche récemment développée par David Bates (qui reprend également la notion de soft power dans son Normans and Empire, paru en 2013). Pour étayer son argumentation, l'auteure décrit cette politique de conversion telle qu'elle avait été conçue et menée par Charlemagne et sa cour, étudiant les rituels de soumission imposés aux païens et la fonction idéologique de " l'amour » chrétien dans le projet d'unification des peuples de l'empire et d'atténuation de la violence de la conquête (p. 29). Ensuite, elle tente de retracer la continuité de cette idéologie dans la 
Normandie unifiée par les premiers ducs, à travers les écrits de Dudon de SaintQuentin. On y trouve en effet nombre de topoï carolingiens pour décrire l'action des ducs, ainsi qu'une vision prophétique du baptême de Rollon comme l'avènement d'un nouvel imperium. Car c'est dans l'interprétation des rêves ou des prophéties, et de manière métaphorique, que de telles conceptions pouvaient s'exprimer. C'est peut-être là, d'ailleurs, une des faiblesses de cet ouvrage : l'impact de ces fictions sur la réalité politique est insuffisamment pris en compte. Politiquement, la hiérarchie traditionnelle des honneurs imposait une réalité contre laquelle la puissance de l'imaginaire et la construction idéologique n'avaient que peu de prise. La popularité de la figure de Charlemagne dans la littérature aurait pu constituer un instrument de mesure de l'efficacité du modèle. Or les textes se l'appropriant sont finalement assez peu nombreux : hormis la Chanson de Roland - dont l'auteure reprend les thèses négligées de ses origines normandes, il faut reconnaitre que l'image de Charlemagne n'est pas très présente. En conséquence, les interprétations proposées de l'idéologie impériale des Anglo-Normands reposent beaucoup sur une analyse de l'implicite. Ainsi, si l'on peut s'accorder sur le fait que le modèle de la royauté carolingienne, comme royauté sacrale, était une référence pour les royautés occidentales, ce modèle était-il vraiment conçu comme nécessairement porteur d'une idéologie impériale ? Le manque de clarification conceptuelle donne l'impression d'une sur-interprétation, et c'est dommage car il y a une véritable idée forte, celle d'une continuité du modèle carolingien chez les Normands jusqu'à la conquête, qui permet d'ouvrir des perspectives stimulantes sur l'idéologie normande en Angleterre. Ce qui manque aussi peut-être dans cette idée d'une continuité, c'est la prise en compte de l'effet de la conquête sur la réactivation du mythe de la translatio imperii. Au contraire, l'auteure semble chercher à minimiser son impact en insistant davantage sur l'idée d'une continuité fluide du modèle impérial entre Charlemagne et les ducs qui s'attachèrent à le poursuivre et le valoriser. Cela l'amène à interpréter l'expansionnisme normand $d u \mathrm{x}^{\mathrm{e}}$ siècle comme un projet d'empire. Mais, dans une Europe féodale fragmentée, cette interprétation n'est pas très convaincante. En réalité, l'assimilation entre idéologie impériale, royauté sacrale et rituels francs gêne l'argumentation plus qu'elle ne la sert. Ainsi, l'auteure établit une continuité directe entre l'idée de conversion politique fondée sur la cohésion des peuples, telle que promue par Charlemagne, et l'idéal civilisé de " courtoisie » envers ses voisins, telle qu'elle est décrite par les auteurs anglais du XII siècle, notamment à travers la figure d'Arthur et de sa cour qui prend du coup des dimensions " impériales» (p. 49). L'auteure offre ainsi, à contre-pied de la définition " moderne » d'impérialisme, une compréhension des politiques impériales de tradition carolingienne, comme politiques de conversion valorisant les « bonnes coutumes » et l'« amour courtois » plutôt que la domination et les conquêtes militaires. Cette belle idée est cependant appliquée de manière trop superficielle au cas de la " conquête » de l'Irlande après 1171 pour pouvoir s'imposer comme véritable prisme d'interprétation.

4 Le deuxième chapitre analyse la topographie et l'investissement des édifices construits en pierre par une mémoire associée à la grandeur du passé impérial romain et des symboles de la romanitas. Ainsi, l'usage de spolia ou bien de formes comme les arches est vu comme une manière parmi d'autres d'exprimer l'idée de translatio. Cela s'applique aussi aux viae regiae, ces voies romaines construites à travers le continent qui permettaient de relier les différents territoires de l'empire. Un détour par les textes bibliques permet de montrer la profondeur des relations entre la route et les devoirs moraux du souverain, qui en avait aussi un usage pratique et politique. Reprenant le 
mythe des Four King's Highways inventé par Henri de Huntingdon, W. M. Hoofnagle montre de manière convaincante que ces voies royales constituaient une marque de la civilisation impériale et de sa puissance unificatrice, ainsi que de l'ordre et de la stabilité conforme aux idéaux des Romains (p.60-64). Huntingdon associe explicitement la domination normande à l'héritage carolingien sur les routes royales, les considérant comme leurs successeurs légitimes. L'auteure doit cependant convenir que les références à la légalité carolingienne ne sont pas toujours évidentes et que, par exemple, l'importance des routes royales en Angleterre constituait aussi et surtout un rappel visuel du passé pré-normand, même si les Anglo-Saxons avaient eux aussi intégré nombre des idéaux carolingiens. Son argument sur l'appropriation normande de la légalité sur les routes royales comme une expression de la translatio imperii évacue cependant tout l'héritage de la bretwalda, qui constituait aussi une tradition impériale britannique, sans doute plus prégnante que celle de Charlemagne, et dont le modèle de référence était tout aussi puissamment byzantin, et donc romain.

Concernant le programme de construction castrale mis en œuvre par les Normands après 1066, l'auteure s'appuie sur le concept de paysage symbolique pour aller à l'encontre d'une vision trop simpliste, mais toujours dominante, des châteaux comme instruments d'oppression militaire, et pour faire valoir leur rôle dans la transformation d'un paysage, réaffirmant l'idée d'un transfert du pouvoir impérial des Romains aux Normands. Réutilisant les sites romains et anglo-saxons qui s'étaient eux-mêmes inscrits dans la continuité antique, les "Normands utilisèrent un style hybride d'architecture non seulement pour souligner leur autorité en Angleterre et leur droit à dominer, mais aussi pour montrer clairement que leur création d'une "nouvelle Rome" à Londres dépassait l'Antiquité et la gloire de l'Empire romain» (p. 73). Comme pour Charlemagne, il s'agissait de surpasser Rome, en créant une nouvelle capitale impériale sur le modèle d'Aix-la-Chapelle. Si l'idée de créer une image de continuité impériale à travers les châteaux, comme symbole d'un "pouvoir de paix», est clairement démontrée, la référence carolingienne apparaît plus évanescente d'autant que des travaux comme ceux de Sandy Heslop ont montré que c'est celle de Constantin qui s'imposait dans bien des cas, et notamment à Colchester. L'idée forte du chapitre, à savoir que l'idée impériale que se faisaient les Normands était une idée non romaine, et donc carolingienne, postule ainsi de manière trop réductrice le modèle carolingien comme le seul modèle alternatif au modèle romain.

6 Le dernier chapitre, enfin, est consacré à la chasse royale et la législation sur les forêts. Il s'agit de montrer que la confiscation des forêts en Angleterre après la conquête reposait sur des principes d'inviolabilité et des enjeux économiques semblables à ceux édictés par Charlemagne. L'auteure reconnaît d'emblée que " tracer l'influence de la royauté carolingienne sur les pratiques ducales normandes, n'est pas, malheureusement, un exercice qui repose sur l'évidence des traces écrites » (p. 91). Elle fait donc reposer son interprétation sur des références implicites en enquêtant sur l'articulation entre la conservation des pratiques régaliennes sur les forestis et leurs associations à l'idée d'une autorité « impériale ». Mais si la chasse était effectivement une pratique permettant au roi d'affirmer sa domination et sa supériorité matérielle, elle n'était pas un monopole royal. Guillaume le Conquérant étendit certes ses forêts aux dépends de l'aristocratie, mais sans l'en déposséder totalement, et on a l'impression ici que l'usage du terme " impérial » sert à qualifier une suprématie dont pourrait se revendiquer la majorité des royautés post-carolingiennes, ce qui est une interprétation possible, mais qui affaiblit alors la force du propos général. L'idée que la 
forêt serait le locus amoenus de la cour se retrouve dans la littérature comme dans la documentation pratique (Geffrei Gaimar, Marie de France) mais de là en déduire son caractère curial et donc impérial est plus difficile à suivre, d'autant que la chasse était une pratique de domination et de contrôle de l'espace partagée par toute l'aristocratie.

7 En conclusion, l'auteure insiste sur la place de la figure de Charlemagne dans deux poèmes produits ou chantés dans le contexte de la conquête : la Chanson de Roland et le Roman des Franceis d'André de Coutances. Si la figure de Charlemagne servit surtout à légitimer la création d'un empire transmanche issu de la conquête, elle doit être également considérée dans son contexte culturel et notamment dans celui de l'essor d'un sentiment pro-anglais qui se cristallise à travers la conflictualité incessante avec le roi de France. Ainsi, l'idée d'Englishness devrait être considérée comme le produit de l'imagination et de l'expérience anglo-normande bien plus que comme une réaction à celles-ci.

Il s'agit donc d'un ouvrage riche par sa démarche et ses idées qui ouvrent des pistes d'interprétations nouvelles et stimulantes. Cependant l'imaginaire impérial de la royauté normande peine à se réduire à la seule figure de Charlemagne, qui ne synthétisait pas, à mon avis, la multitude des conceptions de l'empire existant au $\mathrm{XII}^{\mathrm{e}}$ siècle. La complexité de cette idée d'empire aurait nécessité, pour davantage de clarté, une définition plus fine, la distinguant de celle de royauté sacrale ou de suprématie territoriale, ou de ce qu'on pourrait appeler la " souveraineté », un concept qui s'élabore au cours du XII siècle en reprenant précisément ce vocable d'imperium.

\section{AUTEURS}

\section{FANNY MADELINE}

Fondation Thiers - LAMOP-UMR 8589 